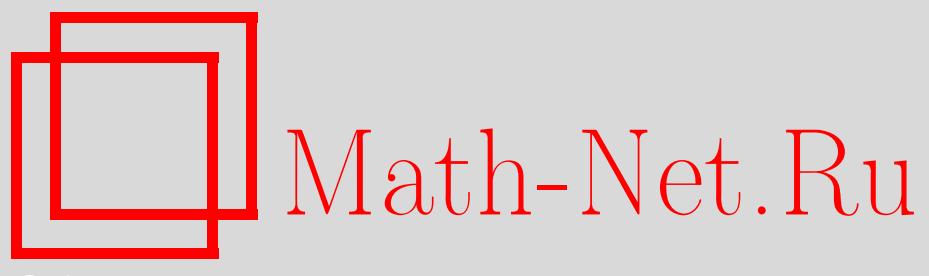

Л. К. Кусаинова, А. Ж. Монашова, А. А. Шкаликов, Асимптотика собственных значений несамосопряженного дифференциального оператора второго порядка на оси, Матем. заметки, 2013, том 93, выпуск 4, 630-633

DOI: https://doi.org/10.4213/mzm10173

Использование Общероссийского математического портала Math-Net.Ru подразумевает, что вы прочитали и согласны с пользовательским соглашением http://www . mathnet.ru/rus/agreement

Параметры загрузки:

IP : 52.90 .164 .192

26 апреля 2023 г., 16:07:40

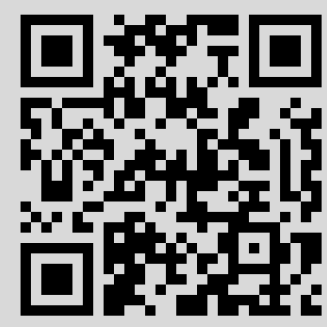




\section{Асимптотика собственных значений несамосопряженного дифференциального оператора второго порядка на оси}

\section{Л. К. Кусаинова, А. Ж. Монашова, А. А. Шкаликов}

В работе изучается оператор, порожденный в пространстве $H=L_{2}(\mathbb{R})$ дифференциальным выражением

$$
l(y)=-y^{\prime \prime}+p(x) y^{\prime}+q(x) y, \quad x \in \mathbb{R},
$$

где $p$ и $q$ - комплекснозначные непрерывные функции. Представим $q(x)$ как

$$
q(x)=q_{0}(x)+i q_{1}(x),
$$

где $q_{0}(x)=\operatorname{Re} q(x), q_{1}(x)=\operatorname{Im} q(x)$, и далее будем полагать выполненными следующие условия:

(i) $\inf _{x \in \mathbb{R}} q_{0}(x)=c>-\infty$;

(ii) $\lim _{x \rightarrow \pm \infty} \int_{x}^{x+a} q_{0}(t) d t=\infty \quad$ при любых $a>0$;

(iii) $\lim _{x \rightarrow \pm \infty} \frac{q_{1}(x)}{q_{0}(x)-c+1}=\lim _{x \rightarrow \pm \infty} \frac{p(x)}{\sqrt{q_{0}(x)-c+1}}=0$.

Обозначим через $L_{0}$ дифференциальный оператор в $H=L_{2}(\mathbb{R})$, определенный дифференциальным выражением

$$
l_{0}(y)=-y^{\prime \prime}+q_{0}(x) y, \quad x \in \mathbb{R},
$$

с областью определения

$$
\mathscr{D}\left(L_{0}\right)=\left\{y \in H: y \in W_{2, \mathrm{loc}}^{2}, l_{0}(y) \in H\right\} .
$$

Здесь $W_{2, \text { lос }}^{2}$ - пространство всех функций $f$ таких, что $f \in W_{2}^{2}(\Delta)$ на любом отрезке $\Delta \subset \mathbb{R}, W_{2}^{2}(\Delta)$ - пространство Соболева. Оператор $L_{0}$ определен на этой области корректно и является полуограниченным. Более того, из теоремы Левинсона [1; VII, §23.6] следует, что $L_{0}$ является самосопряженным оператором, а из условий (i), (ii) и теоремы Молчанова [1; VII, §24.5] следует, что $L_{0}$ имеет дискретный спектр.

Далее, не ограничивая общности, будем считать, что $c>0$ в условии (i) (в противном случае сделаем замену спектрального параметра). Тогда $L_{0}$ равномерно положителен и порождает шкалу компактно вложенных $\left(H_{\alpha} \hookrightarrow H_{\beta}\right.$ при $\left.\alpha>\beta\right)$ гильбертовых пространств $H_{\theta},-\infty<\theta<+\infty$. По определению $H_{0}=H$; при $\theta>0$ полагаем $H_{\theta}=\mathscr{D}\left(L_{0}^{\theta / 2}\right)$ (область определения $L_{0}^{\theta / 2}$ ) с нормой $\|y\|_{\theta}=\left\|L_{0}^{\theta / 2} y\right\|_{0}$, где $\|\cdot\|_{0}=\|\cdot\|_{H}$. При $\theta<0$ пространство $H_{\theta}$ определяем как замыкание $H$ по норме $\|y\|_{\theta}=\left\|L_{0}^{\theta} y\right\|_{0}$. Непосредственно из определения пространств $H_{\theta}$ следует, что $L_{0}$ изоморфно переводит пространство $H_{\theta+2}$ в $H_{\theta}$. Более того, оператор $L_{0}$, рассматриваемый как оператор в $H_{\theta}$ с областью определения $\mathscr{D}\left(L_{0}\right)=H_{\theta+2}$, является самосопряженным и во всех пространствах $H_{\theta}$ имеет один и тот же (дискретный) спектр (см. [2], [3]). В частности, $L_{0}$ с областью определения

Работа первого автора выполнена при поддержке МОН РК по направлению “Фундаментальные исследования в области естественных наук". Работа третьего автора выполнена при поддержке Российского фонда фундаментальных исследований (грант № 10-01-00423).

DOI: $10.4213 / \mathrm{mzm} 10173$ 
$\mathscr{D}\left(L_{0}\right)=H_{-1}$ является самосопряженным оператором в пространстве $H_{-1}$, являющимся двойственным к $H_{1}$. Норма в $H_{1}$ определяется как замыкание квадратичной формы $\left(L_{0} y, y\right)$ и задается равенством

$$
\|y\|_{1}^{2}=\left\|L_{0}^{1 / 2} y\right\|^{2}=\left(y^{\prime}, y^{\prime}\right)+\left(q_{0} y, y\right)=\left\|y^{\prime}\right\|_{0}^{2}+\left\|q_{0}^{1 / 2} y\right\|_{0}^{2} .
$$

Теорема 1. Пусть выполненъ условия (i)-(iii). Тогда

а) оператор $L_{1}$, определенный равенством $L_{1} y=p(x) y^{\prime}+i q_{1}(x) y$, корректно определен на $D\left(L_{0}\right)=H_{1}$ и действует, как компактный оператор из $H_{1}$ в $H_{-1}$;

b) оператор $L=L_{0}+L_{1}$ корректно определен в пространстве $H_{-1}$ (с областъю определения $\left.\mathscr{D}(L)=H_{1}\right)$ и имеет дискретный спектр; при любом $\varepsilon>0$ этот спектр асимптотически (т.е. за исключением конечного числа собственных значений) лежит в угле $|\arg \lambda|<\varepsilon ;$ сужение этого оператора на пространство $H=L_{2}(\mathbb{R})$ имеет область определения

$$
\mathscr{D}(L)=\left\{y \in H_{1}: y \in W_{2, \mathrm{loc}}^{2}, l(y) \in L_{2}(\mathbb{R})\right\}
$$

при этом спектры оператора $L$ в пространствах $H_{-1}$ и в $H$ совпадают.

ДокАЗАтЕЛЬство теоремы 1 получается на следующем пути. Из компактности оператора $L_{0}^{-1}$ в пространстве $H$ (а потому во всех пространствах $H_{\theta}, \theta \in \mathbb{R}$ ) следует компактность вложения $H_{1} \hookrightarrow H_{-1}$. Поэтому утверждение а) теоремы эквивалентно оценке

$$
\left\|L_{1} y\right\|_{-1} \leqslant \varepsilon\|y\|_{1}+M\|y\|_{-1},
$$

справедливость которой нужно установить при любом $\varepsilon>0$ с постоянной $M=M(\varepsilon)$. Доказательство этой оценки проводится с помощью условия (iii) и равенства

$$
\left\|L_{1} y\right\|_{-1}=\sup _{\|f\|_{1}=1}\left|\left(L_{1} y, f\right)\right|=\sup _{\|f\|_{1}=1}\left(\int_{|x|>a}+\int_{|x|<a}\right) L_{1} y(x) \overline{f(x)} d x,
$$

где $a=a(\varepsilon)$ - достаточно большое число. Каждый из интегралов в правой части этого равенства оценивается отдельно.

Для доказательства утверждения b) заметим, что утверждение а) теоремы 1 равносильно следующему: оператор $L_{1}$ является $L_{0}$-компактным в пространстве $H_{-1}$, т.е. произведение $L_{1} L_{0}^{-1}$ является компактным оператором в $H_{-1}$. Тогда дискретность спектра оператора $L$ в пространстве $H_{-1}$ и его асимптотическая локализация в произвольно малых секторах $|\arg \lambda|<\varepsilon$ следует из теоремы Келдыша о компактных возмущениях (см. [4; гл. 5 , $\S 11])$. Далее, воспользуемся равенством

$$
L=L_{0}^{1 / 2}(1+S) L_{0}^{1 / 2}, \quad S=L_{0}^{-1 / 2} L_{1} L_{0}^{-1 / 2}
$$

Можно считать, что оператор $L$ обратим в пространстве $H_{-1}$, в противном случае нужно сделать сдвиг спектра. Тогда оператор $1+S$ обратим в $H$, причем из утверждения а) следует, что $S$ компактный в $H$. Таким образом, из (1) получаем, что $L^{-1}$ компактный в $H$, а потому $L$ имеет дискретный спектр. Доказательство совпадения дискретных спектров оператора $L$ в пространствах $H$ и $H_{-1}$ имеется в работе [3].

На интервале $(c,+\infty)$ (здесь $c$ - число, фигурирующее в условии $(\mathrm{i}))$ определим функцию

$$
M(\lambda)=\frac{1}{\pi} \int_{\lambda \geqslant q_{0}(x)} \sqrt{\lambda-q_{0}(x)} d x .
$$

Эта функция участвует в формулах Карлемана-Тичмарша, устанавливающих асимптотику собственных значений самосопряженных дифференциальных операторов второго порядка (см. [5]). Очевидно, $M(\cdot)$ монотонно строго возрастает на интервале $(c, \infty)$. Поэтому корректно определена обратная к ней функция, которую обозначим через $F(\cdot)$. Через $\left\{\lambda_{n}\left(L_{0}\right)\right\}$ и $\left\{\lambda_{n}(L)\right\}$ обозначим собственные значения операторов $L_{0}$ и $L$ соответственно. Их нумерацию проводим в порядке возрастания модулей и с учетом алгебраической кратности. Далее пишем $\alpha_{n} \sim \beta_{n}$ в случае, если справедливо соотношение $\lim _{n \rightarrow \infty} \alpha_{n} \beta_{n}^{-1}=1$. 
Теорема 2. Пусть найдется число $x_{0} \in \mathbb{R}$ такое, что функиия $q_{0}(\cdot)$ монотонно убъвает на интервале $\left(-\infty,-x_{0}\right)$ и монотонно возрастает на $\left(x_{0},+\infty\right)$. Пусть выполнены равенство $\lim _{|x| \rightarrow \infty} q_{0}(x)=+\infty$ и условие (iii). Тогда для оператора $L=L_{0}+L_{1}$ справедливы соотношения

$$
\lambda_{n}(L) \sim \lambda_{n}\left(L_{0}\right) \sim F(n) .
$$

Сформулируем также аналог теоремы 2 для более сложного случая, когда функция $q_{0}(\cdot)$ не является асимптотически монотонной. В этом случае введем функиию Отелбаева $[6]$

$$
q_{0}^{*}(x)=\inf _{d>0}\left\{d^{-2}: d \int_{x-d / 2}^{x+d / 2} q_{0}(t) d t \leqslant 1\right\} .
$$

Из определения следует $q_{0}^{*}(x)>0$ для всех $x \in \mathbb{R}$, а условие (ii) оказывается эквивалентным условию $q_{0}^{*}(x) \rightarrow \infty$ при $|x| \rightarrow \infty$ (см. [6; I.3]).

Функцию $q_{0}(\cdot)$ назовем $K$-регулярной, если существует непрерывная функция $K: \mathbb{R} \rightarrow$ $[1,+\infty)$ такая, что $\lim _{|x| \rightarrow \infty} K(x)=\infty$ и

$$
\left|\int_{\alpha}^{\beta}\left(q(t)-q^{*}(t)\right) d t\right| \leqslant K^{-1}(x)\left[q_{0}^{*}(x)\right]^{1 / 2}
$$

для всех $\alpha, \beta$ из интервала

$$
\left(x-K(x)\left[q_{0}^{*}(x)\right]^{-1 / 2}, x+K(x)\left[q_{0}^{*}(x)\right]^{-1 / 2}\right) .
$$

Пусть $M^{*}(\cdot)$ - функция, определенная посредством равенства $(2)$ с заменой $q_{0}$ на $q_{0}^{*}$ и постоянной $c$ на $c^{*}=\inf _{\mathbb{R}} q_{0}^{*}$. Пусть $F^{*}(\cdot)$ - обратная к $M^{*}(\cdot)$.

Теорема 3. Пусть выполнены условия (i)-(iii), и пусть функция $q_{0}(\cdot)$ является K-регулярной. Тогда

$$
\lambda_{n}(L) \sim \lambda_{n}\left(L_{0}\right) \sim F^{*}(n) .
$$

Доказательства теорем 2 и 3 получаются с помощью выше приведенной теоремы 1 и асимптотических формул

$$
\lambda_{n}\left(L_{0}\right) \sim F(n) \quad \text { и } \quad \lambda_{n}\left(L_{0}\right) \sim F^{*}(n),
$$

полученных Отелбаевым [6; теорема 1.3 .1 и теорема 1.7.1] для монотонной и немонотонной функции $q_{0}$ соответственно. Используется также теорема Крейна [4; гл. V, теорема 11.3] и соотношение (1), в котором оператор $S$ компактен в пространстве $H$.

Задача об эквивалентности считающих функций собственных значений

$$
n\left(L_{0}, \lambda\right)=\sum_{\lambda_{n}\left(L_{0}\right)<\lambda} 1, \quad n(L, \lambda)=\sum_{\left|\lambda_{n}(L)\right|<\lambda} 1,
$$

является более сложной. Для доказательства эквивалентности этих функций при $\lambda \rightarrow \infty$ необходимо требовать дополнительные условия на регулярность поведения функции $q_{0}(\cdot)$ при $x \rightarrow \pm \infty$ (типа Карлемана-Тичмарша) и тауберовы условия на функцию $M(\cdot)$ (типа Карлемана-Келдыша). При выполнении таких дополнительных условий (см. детали в [5] и [6]) с помощью выше приведенной теоремы 1 и теоремы Келдыша (см. [1; гл. V, §11]) можно получить соотношения

$$
n(L, \lambda) \sim n\left(L_{0}, \lambda\right) \sim M(\lambda) .
$$

Здесь мы не будем формулировать соответствующие дополнительные условия на регулярность функции $q_{0}(\cdot)$ и тауберовы условия на функцию $M(\cdot)$. Но приведем один результат, позволяющий оценить считающие функции $n\left(L_{0}, \lambda\right)$ и $n(L, \lambda)$ при выполнении простых условий на потенциал $q_{0}$.

Теорема 4. Пусть функиия $q_{0}(x)$ асимптотически монотонна при $|x| \rightarrow \infty$ и подчинена оченкам

$$
c_{1}|x|^{\alpha} \leqslant q_{0}(x) \leqslant c_{2}|x|^{\beta} \quad \text { npu } \quad|x|>x_{0},
$$


где $c_{1}, c_{2}, \alpha, \beta>0(\alpha \leqslant \beta)$, а $x_{0}$ - достаточно большое число. Обозначим

$$
a(\alpha):=\frac{1}{\pi} \int_{0}^{1} \sqrt{1-\xi^{\alpha}} d \xi=\frac{1}{\alpha \pi} B\left(\frac{1}{\alpha}, \frac{3}{2}\right)=\frac{\Gamma(1 / \alpha)}{2 \alpha \sqrt{\pi} \Gamma(1 / \alpha+3 / 2)},
$$

где В и Г - бета- и гамма-функиии Эйлера. Тогда при всех $0<\varepsilon<a(\beta)$ справедливы оченки

$$
c_{2}^{-1 / \beta}[a(\beta)-\varepsilon] t^{1 / 2+1 / \beta} \leqslant n\left(L_{0}, t\right) \leqslant c_{1}^{-1 / \alpha}[a(\alpha)+\varepsilon] t^{1 / 2+1 / \alpha}
$$

при достаточно больших $t>t_{0}=t_{0}(\varepsilon)$. Если $\kappa$ тому же выполнено условие (iіi), то оценка (4) справедлива для функиии $n(L, \lambda)$.

ДокАЗАТЕЛЬСтво теоремы 3 получается с помощью равенства

$$
\frac{1}{\pi} \int_{0}^{(\lambda / c)^{1 / \alpha}} \sqrt{\lambda-c x^{\alpha}} d x=c^{-1 / \alpha} a(\alpha) \lambda^{1 / \alpha+1 / 2},
$$

теоремы 2 и вариационной техники для собственных значений самосопряженных операторов. Для доказательства оценки (4) с функцией $n(L, t)$ вместо $n\left(L_{0}, t\right)$ используются теоремы Маркуса-Мацаева [7; гл. $1, \S 8]$ о сравнении спектров для компактных возмущений самосопряженных операторов и представление (1), в котором $S$ - компактный оператор в $H$.

Следствие 1. Если выполнено условие (iii) и оценка (3) при $\alpha=\beta$ (m.e. $\left.q_{0}(x) \asymp x^{\alpha}\right)$, mo

$$
n\left(L_{0}, t\right) \asymp n(L, t) \asymp t^{1 / 2+1 / \alpha} .
$$

Если $q(x) \sim c x^{\alpha}$ и выполнено условие (iii), то

$$
n\left(L_{0}, t\right) \sim n(L, t) \sim c^{-1 / \alpha} a(\alpha) t^{1 / 2+1 / \alpha} .
$$

\section{СПИСОК ЦИТИРОВАННОЙ ЛИТЕРАТУРЫ}

[1] М. А. Наймарк, Линейнъе дифференциалъные операторы, Наука, М., 1969. [2] С.Г. Крейн, Ю.И. Петунин, Е. М. Семенов, Интерполяиия линейных операторов, Наука, М., 1978. [3] А. А. Шкаликов, Тр. сем. им. И. Г. Петровского, 14, Изд-во Моск. ун-та, М., 1989, 140-224. [4] И. Ц. Гохберг, М. Г. Крейн, Введение в теорию линейных несамосопряженных операторов в гильбертовом пространстве, Наука, М., 1965. [5] А. Г. Костюченко, И. С. Саргсян, Распределение собственных значений. Самосопряжсенные обыкновенные дифберенциальные операторы, Наука, М., 1979. [6] М. О. Отелбаев, Оценки спектра оператора Штурма-Лиувилля, Гылым, Алма-Ата, 1990. [7] А. С. Маркус, Введение в спектральную теорию полиномиальных операторных пучков, Штиинца, Кишинев, 1986.

\section{Л. К. Кусаинова}

им. Л.Н.Гумилева, г. Астана

E-mail: leili2006@mail.ru

\section{А. Ж. Монашова}

Евразийский национальный университет

им. Л.Н.Гумилева, г. Астана

E-mail: aygul_monashova@mail.ru

\section{А. А. Шкаликов}

Московский государственный университет им. М. В. Ломоносова

E-mail: ashkalikov@yahoo.com 